\title{
Size-limited penetration of nanoparticles into porcine respiratory mucus after aerosol deposition
}

Xabier Murgia, Paul Pawelzyk, Ulrich F. Schaefer, Christian Wagner, Norbert Willenbacher, and

Claus-Michael Lehr

\section{SUPPORTING INFORMAZION (SI)}

SI 1. Serial dilutions of $100 \mathrm{~nm}, 200 \mathrm{~nm}$ and $500 \mathrm{~nm}$ COOH-modified green-fluorescent nanoparticles (Bang Laboratoriess, stock 1\%) were prepared in triplicate, loaded into a 96-well plate, and their fluorescence intensity was measured with an automated plate reader (excitation $480 \mathrm{~nm}$, emission $520 \mathrm{~nm}$, gain 50; TECAN Infinite 200 PRO). A strong linear correlation was found between nanoparticle concentration (\%) and fluorescence intensity (arbitrary units, a.u.). The orange arrows indicate the particle concentrations (w/v of mucus) used in Fluorescence Recovery after Photobleaching experiments.
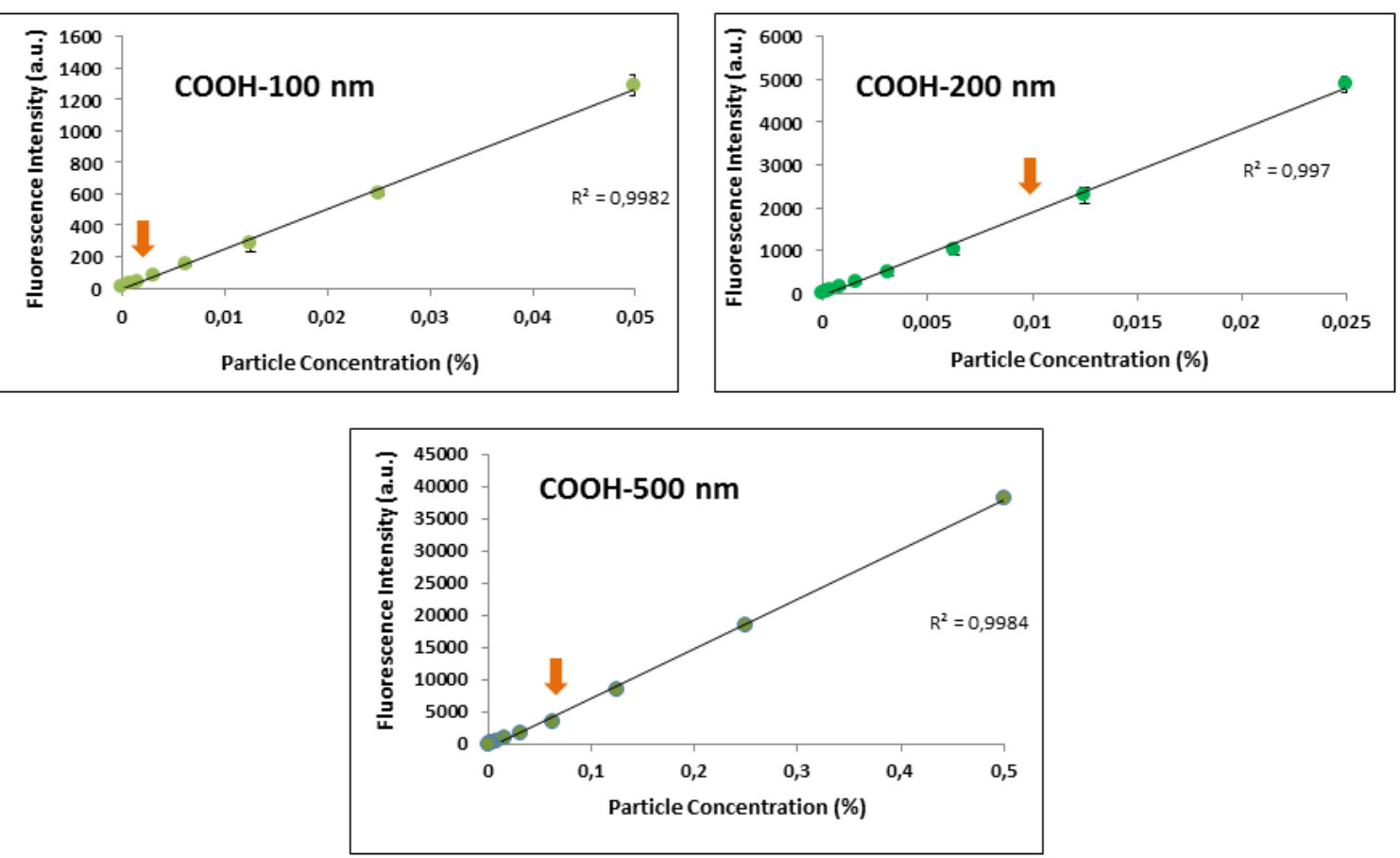
SI 2. Movie 1. Video-recording of $100 \mathrm{~nm} \mathrm{COOH-modified} \mathrm{particles} \mathrm{in} \mathrm{native} \mathrm{porcine} \mathrm{pulmonary}$ mucus. Pictures were taken with an inverted fluorescence microscope (AxioObserver D, Zeiss, Germany) with a Fluar 100x objective including an oil-immersion lens with a numerical aperture of 1.3. at a resolution of $0.062 \mu \mathrm{m}$ per pixel and a frame rate of 50 frames per second. The field of view of the camera represents an area of $127 \times 127 \mu \mathrm{m}$.

SI 3. Movie 2. Video-recording of $200 \mathrm{~nm} \mathrm{COOH}$-modified particles in native porcine pulmonary mucus. Pictures were taken with an inverted fluorescence microscope (AxioObserver D, Zeiss, Germany) with a Fluar 100x objective including an oil-immersion lens with a numerical aperture of 1.3. at a resolution of $0.062 \mu \mathrm{m}$ per pixel and a frame rate of 50 frames per second. The field of view of the camera represents an area of $127 \times 127 \mu \mathrm{m}$.

SI 4. Movie 3. Video-recording of $500 \mathrm{~nm} \mathrm{COOH-modified} \mathrm{particles} \mathrm{in} \mathrm{native} \mathrm{porcine} \mathrm{pulmonary}$ mucus. Pictures were taken with an inverted fluorescence microscope (AxioObserver D, Zeiss, Germany) with a Fluar 100x objective including an oil-immersion lens with a numerical aperture of 1.3. at a resolution of $0.062 \mu \mathrm{m}$ per pixel and a frame rate of 50 frames per second. The field of view of the camera represents an area of $127 \times 127 \mu \mathrm{m}$.

SI 5. Mean Squared Displacement (MSD, $\mu \mathrm{m}^{2}$ ) of $100 \mathrm{~nm}, 200 \mathrm{~nm}$ and $500 \mathrm{~nm}$ carboxylated nanoparticles in pulmonary pig mucus as a function of time scale (sec). The plots on the top describe the individual trajectories of particles in mucus samples that had been stored at $-20^{\circ} \mathrm{C}$ (Frozen) and gradually thawed before analysis, whereas in the plots below the mucus sample were stored at $4{ }^{\circ} \mathrm{C}$ and experiments were conducted within 12-36 hours after mucus collection (Fresh). A comparable variability was found regarding the individual trajectories of the 
differently-sized nanoparticles, irrespective of the storage method. The red line represents the mean MSD of all the trajectories.
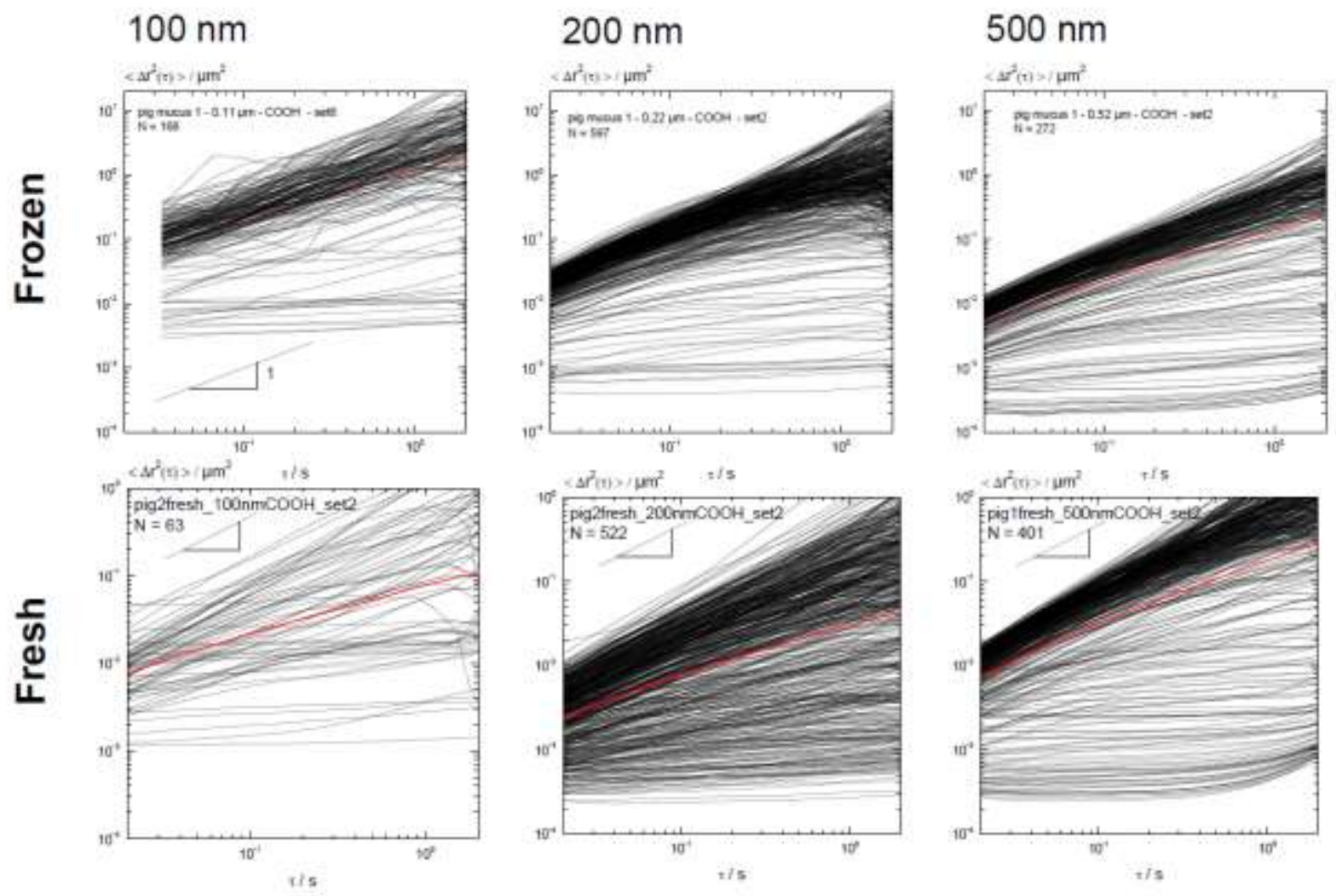